\title{
Design of Non-Coherent CSS Communication Method for Highly Dynamic Scenes
}

\author{
Z.G. Sun \\ College of Information and communication Engineering \\ Harbin Engineering University \\ Harbin, China \\ Science and Technology on Information Transmission and \\ Dissemination in Communication Networks Laboratory \\ Shijiazhuang, China

\section{Y.S. Zheng} \\ College of Information and communication Engineering \\ Harbin Eng ineering University \\ Harbin, China
}

\author{
X.Y. Ning \\ College of Information and communication Engineering \\ Harbin Engineering University \\ Harbin, China \\ Z.M. Chen \\ College of Information and communication Engineering \\ Harbin Eng ineering University \\ Harbin, China
}

\begin{abstract}
High-speed mobile wireless communication system always goes through the doubly selective wireless fading channels, which seriously affects the performance of communication systems. In this paper, a non-coherent chirp spread spectrum (CSS) communication method is proposed based on CORDIC algorithm and differential frequency discriminator. According to the Doppler shift caused by constant and variable speed on the CSS communication method, an inner symbol differential decider is introduced to counteract the Doppler shift in highly dynamic scenes. In the situation of $4 \mathrm{MHz}$ carrier frequency and bit error rate less than $10^{-4}$, computer simulation shows that the proposed method based on CORDIC differential discriminator has a maximum resistance of Doppler shift up to about $150 \mathrm{kHz}$, which is much larger than the coherent method's $30 \mathrm{~Hz}$.
\end{abstract} shift

Keywords-CSS communication; CORDIC algorithm; Doppler

\section{INTRODUCTION}

Chirp signal is a kind of spread spectrum signal, and it is also known as the linear frequency signal or sweep signal. Chirp signal was first used in radar fields with a large time-bandwidth product features for solving a problem in radar ranging between the range and the range resolution. Chirp signal can also be applied in the field of communications. It is called by chirp spread spectrum (CSS) communication for utilizing chirp signals for spread spectrum commun ication system [1].

The demodulation method of the CSS communication can be divided into two types of coherent and non-coherent. The coherent demodulation method performs about $3 \mathrm{~dB}$ SNR higher compared to non-coherent demodulation method. However, the coherent system is relatively complex and it's really strict to the system synchronization [2].

In the situation of the presence of the high-speed relative moving transceivers at both ends of the channel, the Doppler shift cannot be ignored. A non-coherent CSS commun ication system is introduced based on CORDIC algorithm and differential frequency discriminator, and its communication performance and capabilities to resist Doppler shift at highly dynamic scenes are explored, and compared with the coherent CSS communication method.

\section{TECHNICAL FEATURES}

The time domain representation of a typical chirp signal can be written as

$$
s(t)=a(t) \cos \left[2 \pi\left(f_{c} t \pm \mu \frac{t^{2}}{2}\right)\right]
$$

In the eqn (1), ${ }^{a(t)}$ is the signal amp litude, $f_{c}$ is the centre frequency and ${ }^{\mu}$ is the signal chirp rate. At the same time, there is $-T / 2 \leq t \leq T / 2$, $\mathrm{T}$ is the pulse duration period. ' \pm , in formu la above determines the frequency change direction of chirp signal. If it's a ' + ', it indicates that the chirp signal's frequency will increase with time and it is known as up-chirp signal. In contrast, if it is a '-', then it indicates the frequency will decrease with time. And it is called by a down-chirp signal. Up-chirp and down-chirp signal has the same wide bandwidth, time width and chirp rate [3]. The time domain and time-frequency figures of up-chirp and down-chirp signal are shown in fig. 1 and fig. 2 .
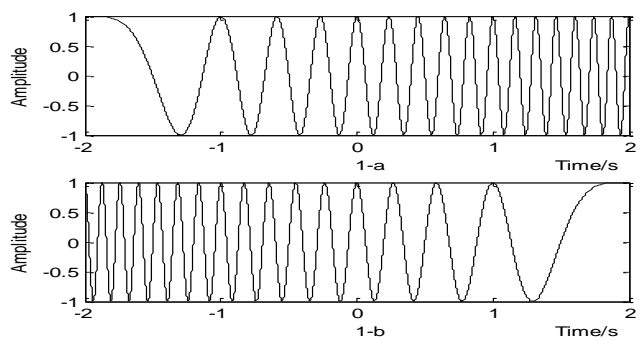

FIGURE I. DIAGRAM OF TIME DOMAIN OF CHIRP SIGNA 

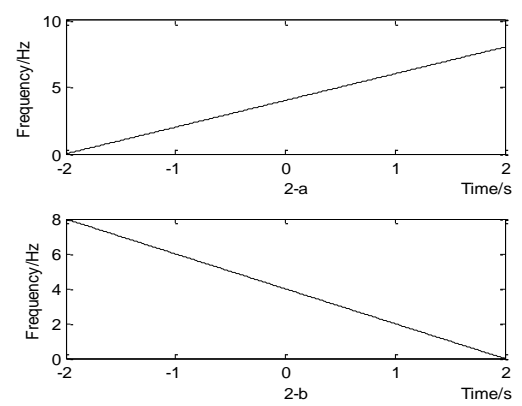

FIGURE II. DIAGRAM OFTIME FREQUENCY OF CHIRP SIGNAL

\section{DESIGN OF NON-COHERENT METHOD}

\section{A. System Model}

The non-coherent CSS communication is made up by the modulator, the channel and the demodulator. ' 1 ' is mapped to the up-chirp signal and ' 0 ' is mapped to the down-chirp signal. The block diagram of the non-coherent CSS communication method is shown in fig. 3 .

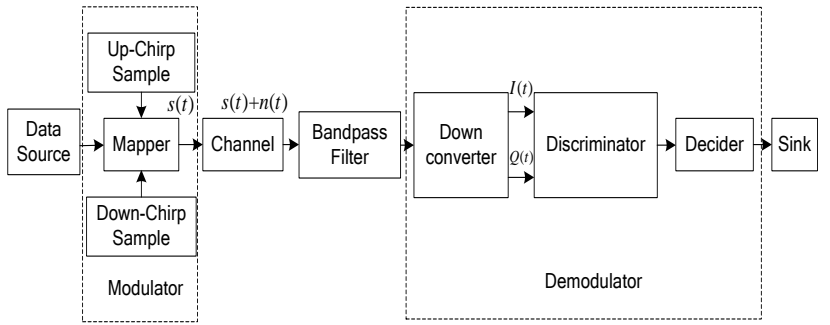

FIGURE III. BLOCK DIAGRAM OF THE NON-COHERENT CSS COMMUNICATION METHOD

\section{B. Design of Discriminator}

In this paper, the output of frequency discriminator directly affects the performance of decider and the bit error rate of the whole system. The frequency discriminator in this article is made up with two parts, the CORDIC phase detector and the first-order differentiator. The block diagram of the discriminator is shown in fig. 4.

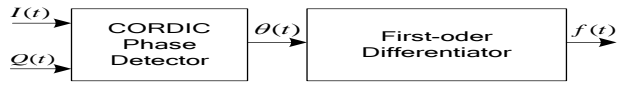

FIGURE IV. BLOCK DIAGRAM OF THE DISCRIMINATOR

If the two baseband signal $I(t)$ and $Q(t)$ are known [4], then the instantaneous phase and frequency of chirp signal can be expressed as

$$
\left\{\begin{array}{c}
\theta(t)=\arctan [Q(t) / I(t)] \\
f(t)=\mathrm{d} \theta(t) / \mathrm{d} t
\end{array}\right.
$$

Assumed $I^{2}(t)+Q^{2}(t)=1$, then it can be further expressed in the discrete form [5]

$$
f(n)=I(n-1) Q(n)-I(n) Q(n-1)
$$

The formula above is the approximate formu la that it can be used to calculate instantaneous frequency $f(n)$ by the two base band signal $I(t)$ and $Q(t)$. However, in order to get the frequency curve, the multiplications are needed. In the actual hardware implementation, the number of multipliers is limited because of the resources. CORDIC algorithm provides a fast and effective way for hardware multiplication, division and arithmetic operations [6][7]. For the discrete digital signal [8], the relationship between the instantaneous phase and frequency under the sampling rate of $T_{s}$ can be expressed as

$$
f(n)=[\theta(n)-\theta(n-1)] /\left(2 \pi \cdot T_{s}\right)
$$

The instantaneous frequency can be calculated by the difference operation of the instantaneous phase.

\section{Design of Decider}

In this paper, the communication environment is highly dynamic scenes, so the modulated signal has a large Doppler shift and the frequency offset is caused by the shift [9]. In the practical systems, the frequency offset rate is a slow transforming process compared with the high sampling frequency and speed symbol rate. Since the amplitude of the signal $^{\mu n T_{s}}$ is symmetric about zero, due to the presence of the Doppler shift, it is equivalent to add a time-varying component on the ideal demodulated signal. Then the demodulated output signal is no longer symmetrical about the zero point.

In this paper, a differential decider inner sy mbol is used to counteract the Doppler shift in highly dynamic scenes [10]. At first, it subtracts the second half cycle of the signal from the first half, and secondly sum the output of subtracting. At last, it compares the result with a value zero. If the value of result is larger, it means the signal transmitted is a '0', or it's a '1' [11]. The diagram of the decider is shown in fig. 5 .

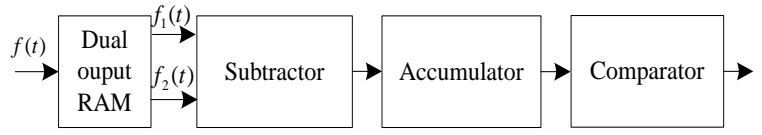

FIGURE V. DIAGRAM OF DECIDER

According to the foregoing, the shift amount of the Doppler shift in a single symbol period may approximately be seen as a uniform frequency offset. So it has no effect on the rate of frequency change within the symbol. Therefore, the inner symbol differential method is proposed to counteract the frequency offset. If the signal in second half cycle is subtracted from the first one, the result can be

$$
\left\{\begin{array}{c}
x_{1}(n)=\mu \times\left(n_{1}-n_{2}\right) T_{s} \\
x_{2}(n)=-\mu \times\left(n_{1}-n_{2}\right) T_{s}
\end{array}\right.
$$

In this eqn (5), there is $0 \leq n_{1} \leq N / 2$ and $N / 2 \leq n_{2} \leq N$. $\mathrm{N}$ is represented as the number of the sampling points in one 
symbol. It's easily visible that the inner symbol differential method can certainly be used to counteract the frequency offset.

\section{SimULATIONS}

\section{A. Added Gaussian White Noise Channel}

TABLE I. CSSCOMMUNICATION SIMULATION PARAMETER SETTINGS

\begin{tabular}{l|l|l|l|l}
\hline $\begin{array}{l}\text { Carrier } \\
\text { frequency( } \\
\mathrm{MHz})\end{array}$ & $\begin{array}{l}\text { Sampling } \\
\text { frequency }(\mathrm{M} \\
\mathrm{Hz})\end{array}$ & $\begin{array}{l}\text { Bandwi } \\
\mathrm{dth} \\
(\mathrm{kHz})\end{array}$ & $\begin{array}{l}\text { Bit } \\
\text { rate } \\
(\mathrm{kbps})\end{array}$ & $\begin{array}{l}\text { Simulati } \\
\text { on times }\end{array}$ \\
\hline 4 & 20 & 700 & 200 & 100000 \\
\hline
\end{tabular}

In order to prove the BER of the non-coherent CSS communication method is correct in this design, MATLAB tools are used to build the non-coherent CSS communication model, and Monte Carlo Method (MCM) is used for simulation

On added Gaussian white noise (AW GN) channel, the BER of non-coherent and coherent CSS communication will be comparative analyzed. Parameter settings are shown in table 1, and simu lation result is shown in fig. 6 .

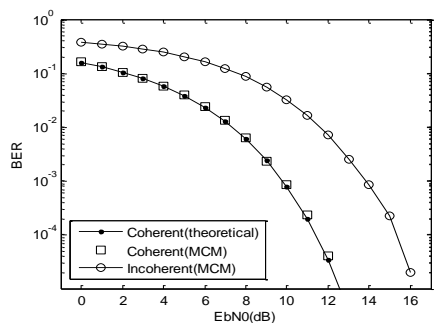

FIGURE VI. BER OF NON-COHERENT AND COHERENT METHOD IN AWGN

According to fig. 6, the BER of coherent method in AWGN is very close to the theoretical value. The non-coherent CSS communication method based on CORDIC differential discriminator is about $3 \mathrm{~dB}$ worse than the BER of coherent one. In the case that $E_{b} / N_{0}$ is greater than $15 \mathrm{~dB}$, the BER of the non-coherent CSS communication method is less than 10-4.

\section{B. Doppler Shift Channel}

Assuming synchronization and Doppler shift exists, the relative movement has constant speed. Assumed that the frequency offset is respectively $20 \mathrm{~Hz}, 30 \mathrm{~Hz}$ and $40 \mathrm{~Hz}$. The BER curve of coherent method is shown in fig. 7. And Set up the frequency offset is respectively $50 \mathrm{kHz}, 100 \mathrm{kHz}$ and $150 \mathrm{kHz}$. The BER curve of non-coherent method is shown in fig. 8 .

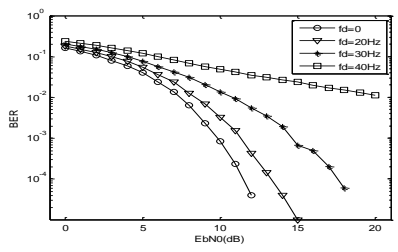

FIGURE VII. BER OF COHERENT CSS MET HODWITH DIFFERENT FREQUENCY OFFSET

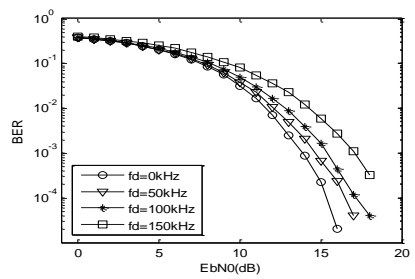

FIGURE VIII. BER OF NON-COHERENT CSS MET HOD WITH DIFFERENT FREQUENCY OFFSET

According to fig. 8, at constant speed, the BER of coherent and non-coherent method both increase with the frequency offset. In order to compare the resistance of Doppler shift of the non-coherent method with the coherent, fig. 7 is compared with fig. 8, and it can clearly be seen that the non-coherent method is much more capable of resisting Doppler shift than the coherent method. In the condition of $4 \mathrm{MHz}$ carrier frequency, the resistance of Doppler shift of the non-coherent method is about $150 \mathrm{kHz}$, while the coherent method is only about $30 \mathrm{~Hz}$.

When the relative speed is variable, the relative speed can be expressed as $f_{d}=A \sin \left(2 \pi f_{n} \cdot t\right)$. Assumed that $f_{n}=20 \mathrm{~Hz}$ and $\mathrm{A}$ is respectively $30 \mathrm{~Hz}, 40 \mathrm{~Hz}$ and $50 \mathrm{~Hz}$, the BER curve of coherent method is shown in fig. 9. Similarly, assumed that $\mathrm{A}=20 \mathrm{~Hz}$ and ${ }^{f_{n}}$ is respectively $20 \mathrm{~Hz}, 40 \mathrm{~Hz}$ and $60 \mathrm{~Hz}$. The simulation results of coherent method is shown in fig. 10. Then assumed that $\mathrm{fn}$ is $80 \mathrm{~Hz}$ and $\mathrm{A}$ is $50 \mathrm{kHz}$, $100 \mathrm{kHz}$ and $150 \mathrm{kHz}$, the BER curve of non-coherent method is shown in fig. 11. Assumed that $A=100 \mathrm{kHz}$ and $f_{n}$ is $10 \mathrm{~Hz}, 30 \mathrm{~Hz}$ and $80 \mathrm{~Hz}$. The simulation results of non-coherent method is shown in fig. 12 .

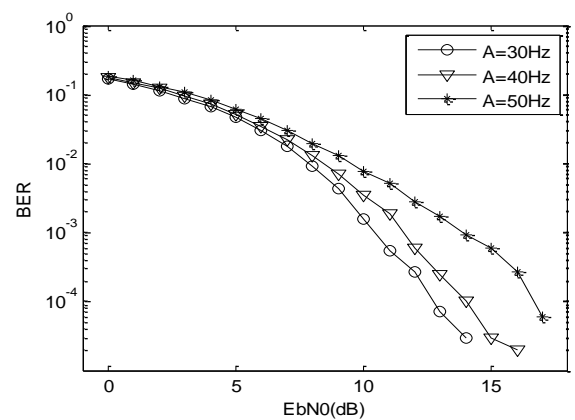

FIGURE IX. BER OF COHERENT METHOD WITH DIFFERENT A

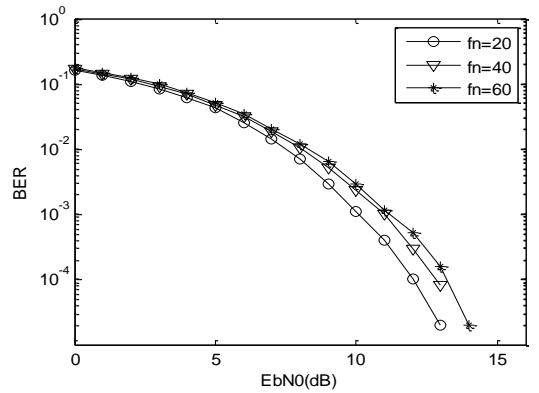

FIGURE X. BER OF COHERENT METHOD WITH DIFFERENT FN 


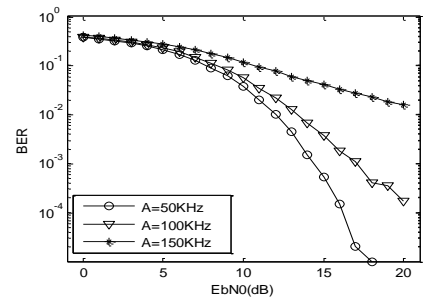

FIGURE XI. BER OF NON-COHERENT MET HOD WITH DIFFERENT MAXIMUM RELATIVE SPEED. FIGURE

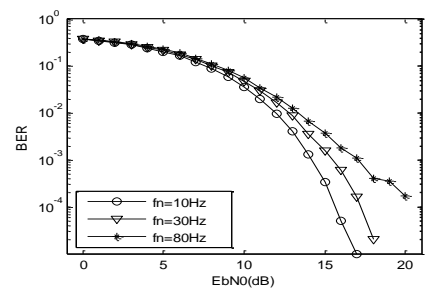

FIGURE XII. BER OF NON-COHERENT METHODWITH DIFFERENT CHANGE RATE

When fig. 9 and 10 are compared with fig. 11 and 12, it can clearly be seen that the non-coherent method is more capable of resisting the Doppler shift caused by variable motion than coherent method.

\section{CONCLUSION}

In conclusion, the non-coherent method's BER performance is $3 \mathrm{~dB}$ less than the ideal coherent method under AWGN channel, but its anti-Doppler shift capability is far stronger than the coherent method, so it is more suitable for transmitting in the highly dynamic scenes.

\section{ACKNOWLEDGEMENTS}

This work was supported by National Natural Science Foundation of China (61101141 and 61401196), the Science and Technology on Information Transmission and Dissemination in Communication Networks Laboratory of China (KX132600013/ITD-U13006) and the Jiangsu Provincial Natural Science Foundation of China (grant No.: BK20140954).

\section{REFERENCES}

[1] LIU, B., Analysis of Ultra Wide-band Transmission Performance System Based on Chirp Spread Spectrum. Modem Electronics Technique, 7, pp. 15-21, 2009.

[2] Springer, A., Gugler, W. \& Huemer, M., Spread Spectrum Communications Using Chirp signals. EUROCOMM 2000 Information Systems for Enhanced Public Safety and Security. Munich Germany, pp. 166-170, 2000

[3] WANG X.W., LI, X. \& FEI, M.R., Anti-noise Performance of Chirp Spread Spectrum System. Communications Technology, 42(3), pp. 36-37, 2009.

[4] Yang, X.N., LOU, C.Y. \& XU, J.L., Software Radio Technology and Application. Beijing Institute of Technology Press: Beijing, pp. 124-125, 2010.

[5] HE, X.H., GUO, H.Z. \& LI, S.J, Digital Realization of FM Demodulation Based on Cross Product Frequency Discriminator. Computer Simulation, 27(3), pp. 353-356, 2010.

[6] Volder, J.E., The CORDIC Trigonometric Computing Technique. IRE Transactions on Computers, 3(8), PP.330-334, 1959.
[7] ZHENG, L., LV, Y.X., XIANG, J.C. \& WANG, L.H., A Digital Frequency Discrimination Technique Based on CORDIC Algorithm. SIGNAL PROCESSING, 19(1), pp.6- 9, 2003.

[8] LU, H.B. \& ZHAO, Y.T, PCM/FM Telemetry Receiving System Based on CORDIC Differential Frequency Demodulation Algorithm. Information and Control, 41(4), pp. 459-464, 2012.

[9] ZHONG X.H., A General Formula of Doppler Shift. College Physics, 14(10), pp. 16-18, 1995

[10] XUN, X.R., ZHANG, J.\& Yao, J., Soft ware Demodulation Method of PCM/DPSK Telemetry Signal. Journal of Telemetry, Tracking and Command, 22(6), pp.9-12, 2001.

[11] WU, H.W, LIU, Y., YANG L.S.\&LI,E.Y., Long-bit Coherent Acquisition Algorithm for Weak GPS Signals Using Differential Decision. Journal of Huazhong University of Science and Technology (Natural Science Edition), 41(7), pp.42-45, 2013. 